\title{
Morphine protects SH-SY5Y human neuroblastoma cells against Dickkopf1-induced apoptosis
}

\author{
KUN-PENG WANG ${ }^{1}$, YU BAI ${ }^{1}$, JIAN WANG ${ }^{1}$ and JIN-ZHEN ZHANG $^{2}$ \\ ${ }^{1}$ Department of Anaesthesiology, The First Hospital of China Medical University, Shenyang, Liaoning 110001; \\ ${ }^{2}$ Department of Anaesthesiology, Shenzhen Hospital, Shenzhen, Guangdong 518000, P.R. China
}

Received January 19, 2014; Accepted September 4, 2014

DOI: $10.3892 / \mathrm{mmr} .2014 .2832$

\begin{abstract}
Morphine is used to relieve pain in patients with cancer in terminal phases. Dickkopf-1 (DKK1), a secreted protein, is a negative regulator of the $\mathrm{Wnt} / \beta$-catenin signaling pathway. Morphine and DKK1 are associated with tumorigenesis. However, to the best of our knowledge, there is no study evaluating the effects of these two factors simultaneously. In the present study, the effects of morphine and DKK1 on neuroblastoma cells in vivo and in vitro were evaluated. To establish the in vitro effects of DKK1 and morphine, human neuroblastoma SH-SY5Y cells were transfected with a DKK1-expressing plasmid and cell migration, apoptosis, migration and invasion were evaluated prior to and following morphine treatment. The results indicated that DKK1 induced apoptosis and inhibited the mobility of neuroblastoma cells and that morphine attenuated these DKK1-induced effects. To evaluate the effects of DKK1 and morphine in vivo, a mouse model of neuroblastoma was established, where mice bearing tumors of native SH-SY5Y cells were injected with DKK1. Tumor size, spatial memory and survival rate were investigated in untreated, DKK1-treated and DKK1+morphine-treated mice. Water maze and T-maze tests were performed, which revealed that DKK1-treated mice exhibited a better memory than DKK1 + morphine-treated mice. The expression of DKK1 in established xenografted tumors was associated with decreased tumor size and an increased survival rate, whereas morphine reversed these effects. Furthermore, it was confirmed that morphine and DKK1 take effect, at least in part, via the $\mathrm{Wnt} / \beta$-catenin signaling pathway. The results of the present study indicate that morphine may protect neuroblastoma cells and thus, it may be used in neuroblastoma patients.
\end{abstract}

Correspondence to: Dr Kun-Peng Wang, Department of Anaesthesiology, The First Hospital of China Medical University, 155 Nanjing North Street, Shenyang, Liaoning 110001, P.R. China E-mail: sykpwang@163.com

Key words: Dickkopf-1, morphine, SH-SY5Y cells, apoptosis, survival rate

\section{Introduction}

Morphine is used to relieve pain in patients with cancer in terminal phases, in order to improve their quality of life (1). Xenograft mouse models were established in previous studies in order to analyze the role of morphine in cancer cells $(2,3)$. Tegeder et al (2) discovered that morphine significantly reduced tumor growth through a p53-dependent mechanism in a mouse model of breast cancer. Harimaya et al (3) demonstrated that morphine inhibited tumor metastasis formation in a mouse model of colon cancer. By contrast, other studies indicated that morphine increased tumor growth $(4,5)$. A further study revealed that morphine enhanced tumor neovascularization by activating vascular endothelial growth factor receptor (6).

The secreted protein Dickkopf-1 (DKK1) is a member of the Dickkopf gene family, which comprises Dickkopf-1, -2, -3 and -4, as well as Dickkopf-3-associated protein, Soggy (7). DKK1 is known to be a negative regulator of the $\mathrm{Wnt} / \beta$-catenin signaling pathway (8). Numerous cellular processes are mediated by Wnt/ $\beta$-catenin signaling, including proliferation, differentiation, survival, apoptosis and cell motility (9). Abnormal DKK1 expression is found in numerous malignant tumors including breast cancer, lung cancer, esophageal carcinoma and hepatocellular carcinoma (10-13). DKK1 is typically silenced in colon cancer cells by DNA hypermethylation, which correlates with the advanced stages of colorectal tumorigenesis (14). Other studies have shown that DKK-1 serves as a diagnostic and prognostic biomarker of hepatocellular carcinomas and osteosarcoma $(15,16)$.

Morphine and DKK1 are associated with tumorigenesis. However, to the best of our knowledge, there has been no study investigating the effects of these two factors simultaneously. The present study therefore aimed to elucidate the effects and interactions of morphine and DKK1 in SH-SY5Y human neuroblastoma cells. Neuroblastoma is a highly malignant pediatric cancer derived from precursor or immature cells of the sympathetic nervous system (17). It is characterized by unexpected clinical behaviors like spontaneous regression or maturation, but also by aggressive progression and poor treatment response (18). In this study, spatial learning and memory were evaluated in mouse models of neuroblastoma using the water maze and T-maze tests. This is the first study to our knowledge to investigate the association between DKK-1 and morphine in human neuroblastoma. Morphine appears to be 
of crucial biological importance in protecting neuroblastoma from the toxicity of DKK1. DKK1 may be used as a novel therapeutic strategy for neuroblastoma.

\section{Materials and methods}

Cell culture. Human neuroblastoma (SH-SY5Y) cells were purchased from the American Type Culture Collection (Manassas, VA, USA). Cells were grown at $37^{\circ} \mathrm{C}$ in medium containing a 1:1 ratio of Eagle's minimum essential medium and Ham's F-12 nutrient mixture (Hyclone, Logan, UT, USA) as well as $10 \%$ fetal bovine serum (FBS; Hyclone) in a humidified atmosphere containing $5 \% \mathrm{CO}_{2}$.

Plasmid transfection and stable cell establishment. The plasmid, phosphorylated enhanced green fluorescent protein (pEGFP)-C1-DKK1 was provided by Dr Wang Rong (China Medical University, Shenyang, China)(19). pEGFP-C1-DKK1 transfections were performed using Lipofectamine ${ }^{\circledR} 2000$ (Invitrogen Life Technologies, Carlsbad, CA, USA) according to the manufacturer's instructions. Twenty-four hours post-transfection with pEGFP-C1-DKK1, cells were selected with G418 (400 $\mu \mathrm{g} / \mathrm{ml}$; Invitrogen Life Technologies) for 10-12 days. Drug-resistant clones were isolated and expanded. All gene expression studies were conducted using pools of colonies $(n=50)$ to avoid clonal bias. The three resulting DKK1-positive cell lines were named D1, D2 and D3.

Immunofluorescence. Transfected cells were washed with phosphate-buffered saline (PBS), fixed in 4\% paraformaldehyde, permeabilized in 1\% Triton X-100 (Beyotime Institute of Biotechnology, Shanghai, China) for 5 min and blocked with $5 \%$ bovine serum albumin (BSA) in PBS containing $0.5 \%$ Triton $\mathrm{X}-100$ for $1 \mathrm{~h}$. DKK1 expression was detected using goat polyclonal immunoglobulin G anti-DKK1 antibody (sc-30782; 1:200; Santa Cruz Biotechnology, Inc., Dallas, TX, USA) for $1 \mathrm{~h}$ at room temperature. Cells were subsequently washed with PBS and incubated with appropriate secondary fluorophore-conjugated antibodies (1:1000) for $1 \mathrm{~h}$ at room temperature. Secondary antibody used for detection of DKK1 was Alexa Fluor ${ }^{\circledR} 594$ donkey anti-goat immunoglobulin G, heavy and light chains (1:200; Invitrogen Life Technologies).

Morphine treatment and cell growth inhibition assays (MTT assay). D1, D2 and D3 cells were treated with a single dose of morphine $(1,5,10$ or $15 \mu \mathrm{M})$. Cells were plated in 96-well plates (1,500 cells/well) and allowed to attach overnight. MTT solution (Sigma-Aldrich, St. Louis, MO, USA) was added (final concentration, $0.5 \mathrm{mg} / \mathrm{ml}$ ) and cells were incubated for $4 \mathrm{~h}$. Next, the cells were lysed using with dimethyl sulfoxide (Sigma-Aldrich). Absorbance was measured at 550-560 nm using a 505 microplate reader (Bio-Rad, Hercules, CA, USA).

Annexin V-fluorescein isothiocyanate (FITC) and propidium iodide (PI) double staining. D1, D2 and D3 cells were left untreated, or were treated with morphine. According to the manufacturer's instructions (Apoptosis Detection kit, KeyGEN, Nanjing, China), cells were washed and resuspended in binding buffer prior to the addition of FITC-labeled Annexin-V and PI for $10 \mathrm{~min}$. Suspensions were immediately analyzed by a FACSCalibur machine (BD Biosciences, Baltimore, MD, USA).

Reverse transcription (RT)-polymerase chain reaction (PCR). mRNA levels of DKK1 were determined by RT-PCR. GAPDH served as the negative control. Total RNA $(20 \mu \mathrm{g})$ was extracted from transfected cells using TRIzol reagent (Invitrogen Life Technologies). Reverse transcription was performed using the RT reaction mix (Promega Corporation, Madison, WI, USA) and the cDNA obtained was used for PCR. The following primers were used for DKK1 and GAPDH were used: Forward, 5'-CTGCATGCGTCACGCTATGT-3' and reverse, 5'-TCCTCGGAAATGATTTTGATCA-3' for DKK1; and forward, 5'-CAGTCAGCCGCATCTTCTTTT-3' and reverse, 5'-GTGACCAGGCGCCCAATAC-3' for GAPDH.

Transwell assay. The migration assay was performed using Boyden chambers $(8-\mu \mathrm{M}$ pore size polycarbonate membrane; Cell Biolabs, San Diego, CA, USA). Cells were resuspended in FBS-free Eagle's minimum essential medium (EMEM; Hyclone) to a concentration of $3 \times 10^{5}$ cells $/ \mathrm{ml}$. The upper chamber was loaded with $100 \mu 1$ cell suspension and the lower chamber was loaded with $600 \mu 1$ EMEM containing $10 \%$ FBS. Following incubation for $12 \mathrm{~h}$ under normal culture conditions, no cells were observed floating in the upper chamber, indicating that the cells had not undergone apoptosis at this time-point. The filter was fixed in $4 \%$ paraformaldehyde (Sigma) and stained with crystal violet (Beyotime, Shanghai, China). The cells on the upper side of the filter were wiped off using a cotton swab. Cells that had migrated to the lower side of the membrane were counted using a light microscope (CX31; Olympus Corporation, Tokyo, Japan). Ten microscopic fields (x400) were randomly selected to count the cells. For the cell invasion assay, the procedure was identical to that outlined above, excluding the Matrigel-coated insert (BD Biosciences).

Stereotaxic surgery and intracerebral administration in the mouse. All animal experiments with were performed according to the guidelines of the China Medical University Ethics Committee (Shenyang, China). Six to eight week-old NU/NU nude mice (1.4-1.8 g) were purchased from Vital River (Beijing, China). The mice were kept at $22^{\circ} \mathrm{C}$ and exposed to a $12 \mathrm{~h}$ light/dark cycle $(6.30 \mathrm{am}-6.30 \mathrm{pm})$ with free access to food and water. All mice received intrastriatal injections of SH-SY5Y cells $\left(1 \times 10^{6}\right.$ in $\left.200 \mu 1\right)$, which were administered $5 \mathrm{~mm}$ below the dura over a 10-min duration with a microsyringe (Hamilton, Reno, NV, USA). The needle was left in place for a further three minutes. The burr hole was sealed with bone wax and the skin incision was closed with 4-0 silk sutures. When the tumor diameters had reached 3-5 mm, the mice were divided randomly into three groups (tumor, DKK1 and DKK1 + morphine groups). The groups were treated with phosphate-buffered saline, a $100 \mu \mathrm{l}$ intratumoral injection of DKK1 $(20 \mu \mathrm{g})$ and $30 \mu \mathrm{l}$ Lipofectamine 2000 (Invitrogen Life Technologies), or DKK1 (20 $\mu \mathrm{g})$ with Lipofectamine $2000(30 \mu \mathrm{l})$ and morphine. Two injections were administered at 9 am and 4 pm every four days. 


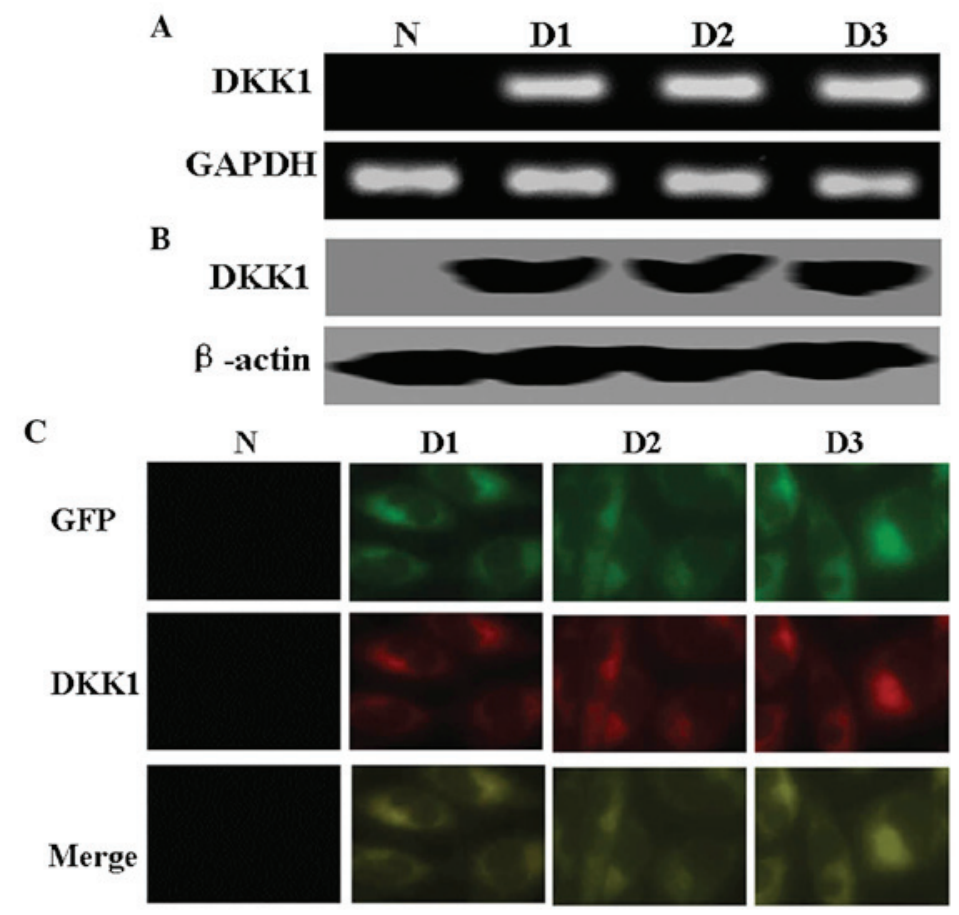

Figure 1. Confirmation of exogenous DKK1 expression in SH-SY5Y cell clones. (A) Polymerase chain reaction analysis of DKK1 messenger RNA expression levels and (B) western blot analysis of DKK1 protein expression levels in SH-SY5Y cells transfected with pEGFP-C1-DKK1. (C) Cells were transfected with pEGFP-C1-DKK1, and immunofluorescence performed with the indicated antibodies (magnification, x200). Immunofluorescence indicated localization of DKK1 and EGFP in DKK1-expressing SH-SY5Y cells. N, untreated SH-SY5Y cells; D1, D2 and D3, DKK1-expressing clones. DKK1, dickkopf-1; EGFP, enhanced green fluorescent protein.

A

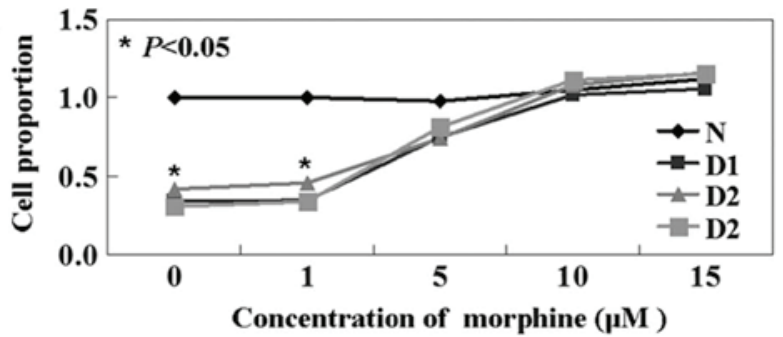

C

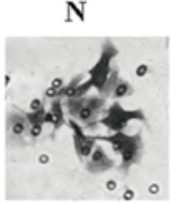

D1

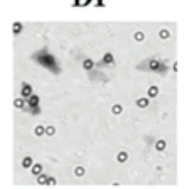

N-M

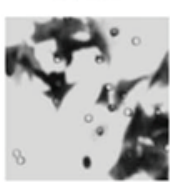

D1-M

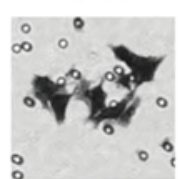

D2

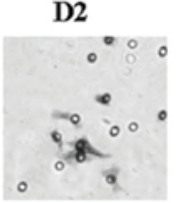

D2-M
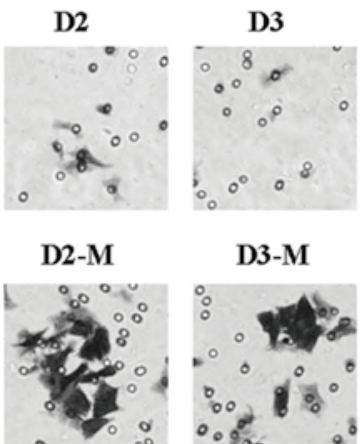

D3-M
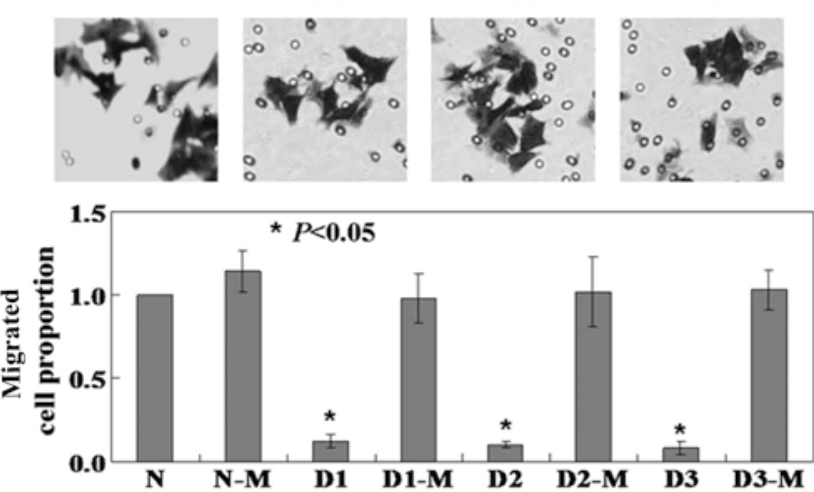

B
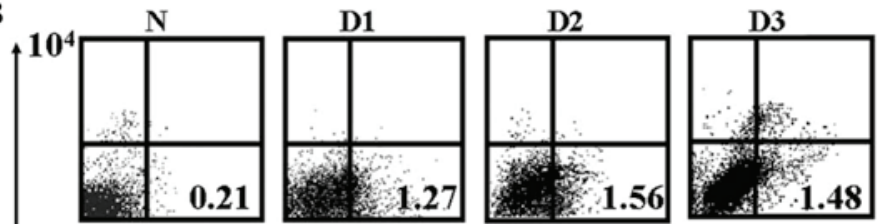

N-M

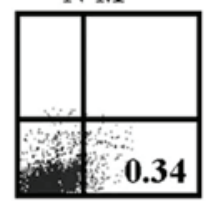

D1-M
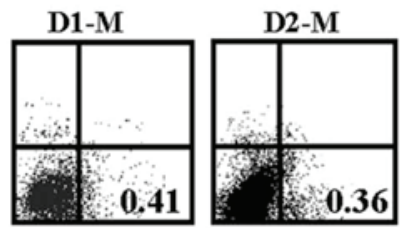

D3-M

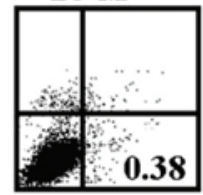

Annexin V-FITC

10

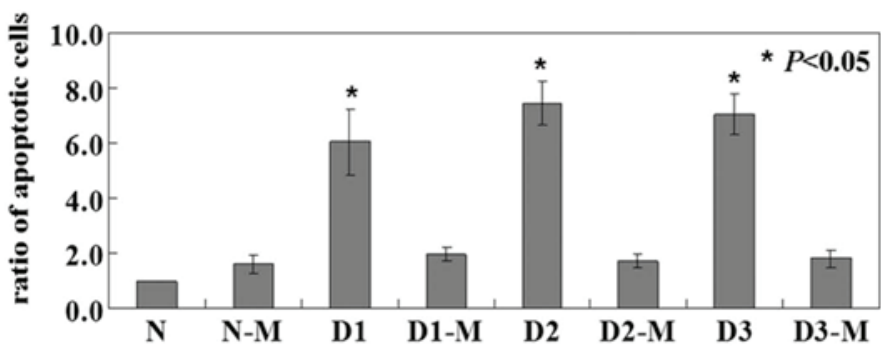

Figure 2. Effects of morphine on DKK1-induced apoptosis in SH-SY5Y cells. (A) Proliferation rate of SH-SY5Y cells was measured using the MTT assay. (B) Proportion of apoptotic cells was determined by double-staining with Annexin-V/fluorescein isothiocyanate and propidium iodide. (C) Transwell assays were performed. Cells that migrated to the bottom side of the membrane were stained and counted (magnification, x400). D1-M, D2-M and D3-M; D1, D2, and D3 DKK1-expressing cells treated with morphine; N, untreated SH-SY5Y cells; FITC, fluorescein isothiocyanate. 

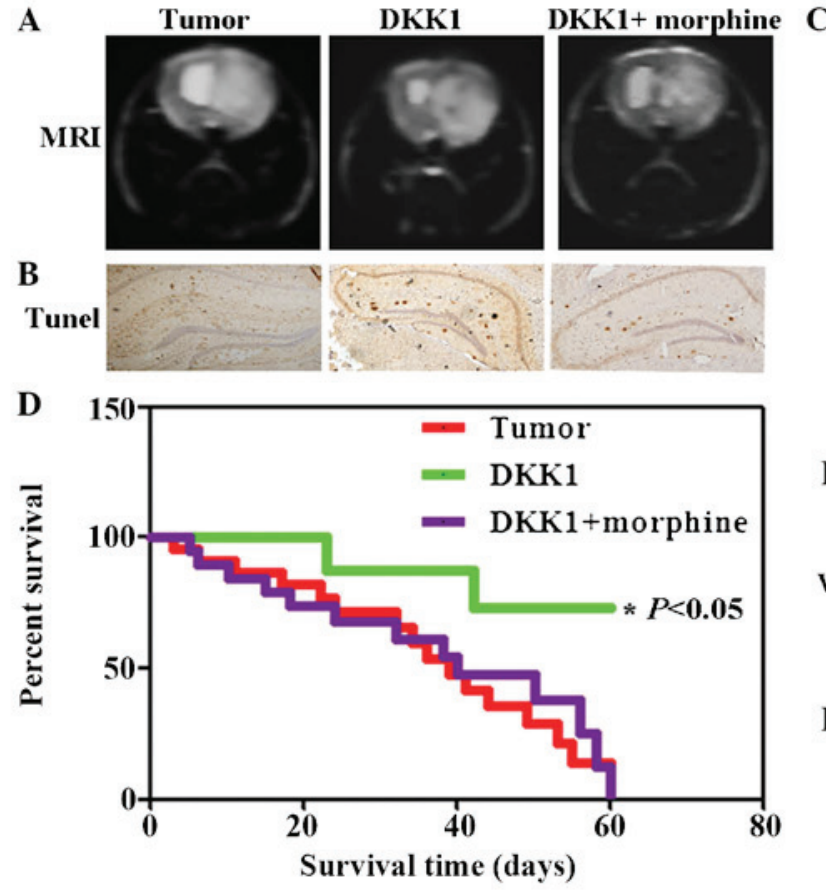

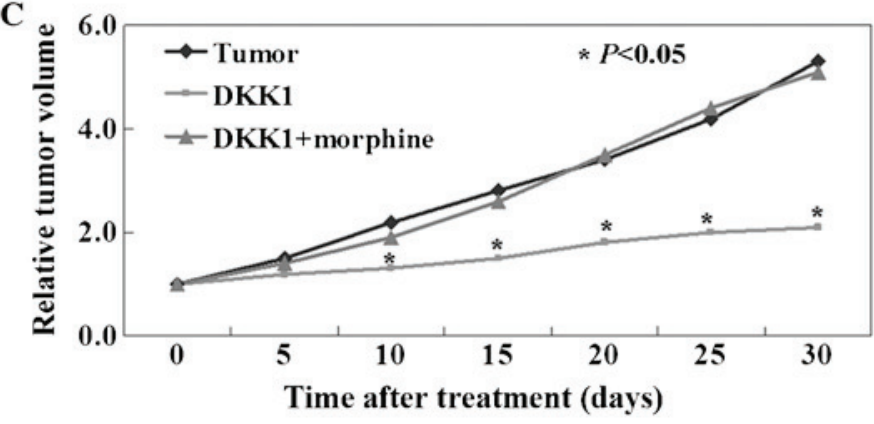

$\mathbf{E}$ Untreated Tumor DKK1 DKK1+morphine

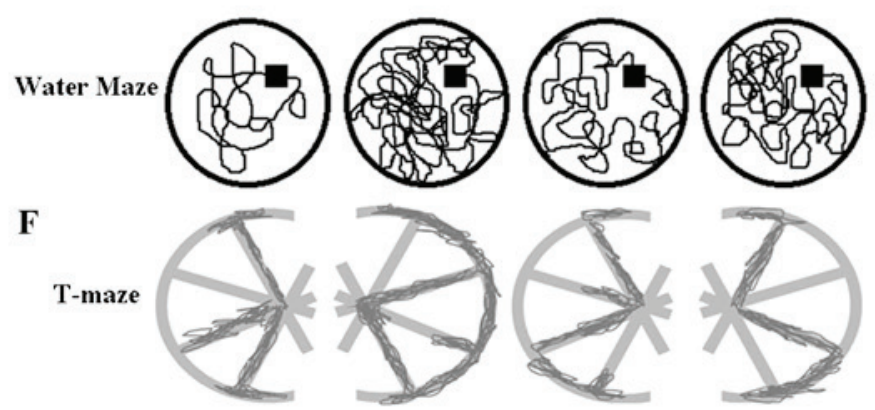

Figure 3. Effects of morphine on DKK1-induced apoptosis in xenograft mouse models. (A) In vivo MRI of tumors in each group. (B) TUNEL staining indicated the roles of morphine in DKK1-induced apoptosis (magnifiction, x100). (C) Tumor volumes of the three groups, sacrificed 30 days following cell-injection (D) Kaplan-Meier survival curves of the groups. (E) Representative spatial trajectory of one mice of each group in the water maze test. (F) Representative spatial trajectory of one mice of each group in the T-maze test. MRI, magnetic resonance imaging; DKK1, dickkopf-1; TUNEL, terminal deoxynucleotidyl transferase-mediated deoxyuridine triphosphate nick-end labeling.

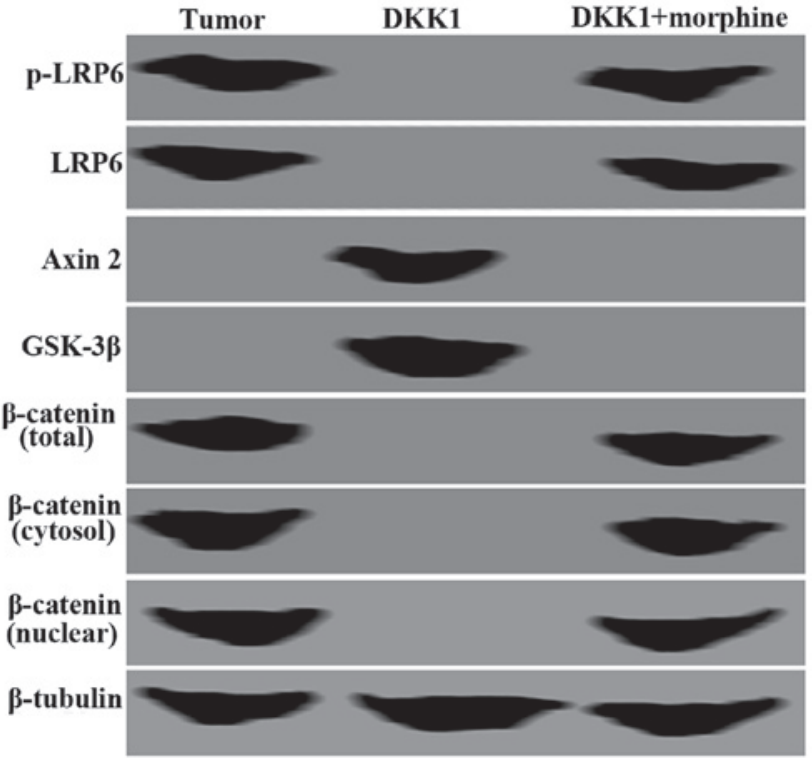

Figure 4. Changes in the Wnt/ $\beta$-catenin signaling pathway. Cellular protein was isolated from SH-SY5Y cells and western blot analysis was performed to detect LRP6, p-LRP6, Axin2, GSK-3 $\beta$, and $\beta$-catenin protein expression levels using the respective specific antibodies. $\beta$-tubulin was used as an internal control. p-LRP6, phosphorylated low-density lipoprotein receptor-related pro-

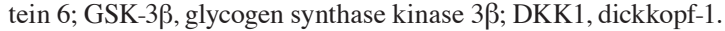

Water maze test. Behavioral observations for spatial learning and memory were made in the water maze, a modification of the standard version of the Morris test (20). Animals were trained to find a hidden platform in a swimming pool.
The circular white pool was filled to a depth of $30 \mathrm{~cm}$ with $23^{\circ} \mathrm{C}$ water. The pool was located in a testing room which contained numerous objects that could be used by the mice for spatial orientation. The position of the cues remained unchanged throughout the period of testing. Each trial was started by placing a mouse facing towards the wall of the pool at the start point. The sequence of the starting positions was randomly selected and changed each day. The trial was terminated when the animal mounted the hidden platform. Ten mice were used in each experiment and each experiment was performed in triplicate.

T-maze test. Two identical four-arm radial mazes arranged in a single large wagon-wheel structure $(120 \mathrm{~cm}$ outer diameter; width of arms, $6 \mathrm{~cm}$ ) were used for the T-maze test. The center platform was common to the two mazes. One movable transparent wall on an outer arm and eight transparent doors around the center allowed the selection of a specific configuration for each maze. Each mouse was initially placed at one end of the trajectory and required to navigate through the correct arms in order to reach the opposite end and retrieve chocolate sprinkles. The mouse subsequently had to return to the starting position, where it received chocolate sprinkles again, to initiate a novel trial. Each recording session began with a one-hour resting period in a small, separate box. The mouse was then trained for 15 min on the first maze, allowed to rest for one hour, then trained for $15 \mathrm{~min}$ on the second maze and allowed to rest for another hour. One maze was selected as the test maze and the other as the control maze; the temporal order and physical location of the test maze on 
the first day was randomized across mice. For each mouse, the test and control mazes remained unchanged for the duration of training. The injection of DKK1 and/or morphine was always performed during the resting period following maze $\mathrm{T}$, and the order of the mazes was alternated each day for 8-10 days. On the last planned experimental day, no stimulation was applied. Recordings had to be prematurely halted following three days of testing for one mouse due to technical reasons.

Animal magnetic resonance imaging (MRI). For the MRI (Aspect M2; Winsun China Ltd., Hong Kong, China), anesthesia was induced with $4 \%$ isoflurane and maintained with $2.7 \%$ isoflurane in $69 \% \mathrm{~N}_{2} \mathrm{O}$ and $30 \% \mathrm{O}_{2}$ using a vaporizer. MRI sessions were performed 10 and 11 days following cell inoculation when tumors were $2-3 \mathrm{~mm}$ in diameter.

Terminal deoxynucleotidyl transferase-mediated deoxyuridine triphosphate nick-end labeling (TUNEL). Tumors were resected and the tumor volume was determined. Tumor biopsies were immediately fixed in $10 \%$ neutral-buffered formalin containing phosphatase inhibitors ( $\mathrm{NaF}$ and $\mathrm{Na}_{3} \mathrm{VO}_{4}$; Beyotime Institute of Biotechnology) prior to the preparation of paraffin-embedded sections. Apoptosis was measured using immunohistochemistry in paired tumor samples by TUNEL.

Preparation of nuclear and cytoplasmic protein extracts. Nuclear and cytoplasmic protein fractions were isolated at the indicated time-points using a CelLytic ${ }^{\mathrm{TM}}$ NuCLEAR $^{\mathrm{TM}}$ Extraction kit (Sigma), according to the manufacturer's instructions. Protein concentrations were determined using a bicinchoninic acid protein assay with BSA used as a standard (Pierce Biotechnology, Inc., Rockford, IL, USA).

Western blot analysis. Western blot analysis was performed using cell extracts from SH-SY5Y cells transfected with pEGFP-C1-DKK1. Cell extracts were resolved using SDS-PAGE and subsequently transferred onto nitrocellulose membranes. Membranes were developed and visualized using enhanced chemiluminescence (Pierce Biotechnology, Inc., Rockford, IL, USA). Primary antibodies used included: Rabbit polyclonal IgG anti-low-density lipoprotein receptor-related protein 6 (LRP6) (sc-15399; Santa Cruz Biotechnology, Inc.), mouse polyclonal IgG anti-phosphorylated-LRP6 (Ser1490) (2568; Cell Signaling Technology, Beverly, MA, USA), rabbit monoclonal IgG anti-Axin2 (5863; Cell Signaling Technology), mouse monoclonal IgG anti-glycogen synthase kinase $3 \beta$ (GSK-3 $\beta$; sc-81462; Santa Cruz Biotechnology, Inc.), mouse monoclonal IgG anti- $\beta$-catenin (610154; BD Biosciences), and mouse monoclonal IgG anti- $\beta$-tubulin (T5201; Sigma-Aldrich).

Statistical analysis. All values are presented as the mean \pm standard error of the mean. Student's paired t-test was used to identify statistical significances. Kaplan-Meier survival plots were generated and comparisons were made with log-rank statistics. $\mathrm{P}<0.05$ was considered to indicate a statistically significant difference between values. Statistical analyses were performed using GraphPad PRISM 4 software (GraphPad Software Inc., San Diego, CA, USA).

\section{Results}

DKK1-expressing $\mathrm{SH}-\mathrm{SY} 5 Y$ cell lines. SH-SY5Y cells were transfected with pEGFP-C1-DKK1, and EGFP-expressing SH-SY5Y cells were selected for further study. As shown in Fig. 1A and B, the results of RT-PCR and western blot analysis confirmed exogenous expression of DKK1 in SH-SY5Y cells following transfection. Immunofluorescence analysis revealed the localization of DKK1 and EGFP in DKK1-expressing SH-SY5Y cells (Fig. 1C).

Effects of morphine on DKK1-expressing SH-SY5Y cells. The function of DKK1 on SH-SY5Y cells was established. The results of the MTT assay indicated that the proliferation rate of cells in the D1, D2 and D3 groups was significantly lower than that of untreated cells $(\mathrm{P}<0.05$; Fig. 2A). The ratio of apoptotic cells in the D1, D2 and D3 groups was higher than that of the untreated cells, established by Annexin-V and PI double-staining $(\mathrm{P}<0.05$; Fig. 2B). The effect of DKK1-expression on the migration of SH-SY5Y cells was determined by Transwell assay. Migration was significantly inhibited in D1, D2 and D3 cells compared to that of untreated cells $(\mathrm{P}<0.05$; Fig. 2C). Subsequently, the effect of morphine on DKK1-expressing SH-SY5Y cells was determined. Following morphine treatment, D1, D2 and D3 groups exhibited a higher proliferation rate, lower apoptotic rate and greater mobility than untreated DKK1-expressing cells ( $\mathrm{P}<0.05$; Fig. 2).

Anti-tumor activity of morphine and DKK1 in a mouse model. Neuroblastoma mouse models were used in order to assess the in vivo tumorigenic or anti-tumor effects of DKK1 and morphine. Fig. 3A exhibits representative T2-weighted MRIs of the mouse tumor model using the spin-echo pulse sequence method, prior to and following injection of DKK1 or DKK1+morphine. The results of the TUNEL assay indicated a higher apoptotic rate of tumor cells in mice following DKK1 treatment and a lower apoptotic rate of cells in mice following DKK1+morphine treatment (Fig. 3B). Correspondingly, the volumes of DKK1-treated tumors were lower than those of untreated and those of DKK1+morphine-treated tumors $(\mathrm{P}<0.05$; Fig. 3C). In addition, the survival rate of mice with tumors treated with DKK1 was significantly improved $(\mathrm{P}<0.05)$. Mice in the untreated and DKK1+morphine-treated groups died at the end of the experiment (Fig. 3D). Furthermore, the acquired and retained memory of the mice with DKK1 or morphine treatment was examined using the water maze. It was confirmed that the DKK1-treatment group demonstrated a superior memory in comparison to the other groups (Fig. 3E). The number of Start-to-Finish trajectories completed by each mouse on the T-maze test as a function of time was evaluated. Significantly shorter trajectories were observed in the mice with DKK1 treatment than in those in the untreated and DKK1+morphine-treated groups (Fig. 3F).

Morphine mitigates DKK1-induced apoptosis in SH-SY5Y cells. As indicated in Fig. 4, a decrease in p-LRP6 and LRP6 expression and an increase in Axin 2 and GSK-3 $\beta$ expression in SH-SY5Y cells with DKK1 expression was observed. By contrast, the altered expression levels of these proteins were reversed following DKK1+morphine treatment (Fig. 4). 
Total, cytosolic and nuclear $\beta$-catenin protein expression was lost in SH-SY5Y cells following DKK1 treatment. However, the expression of these proteins was restored following DKK1+morphine treatment (Fig. 4). $\beta$-tubulin was used as an internal control.

\section{Discussion}

Morphine, a potent natural opiate, is commonly used for the treatment of patients with severe tumor-associated pain. Despite extensive research, it remains to be elucidated whether morphine directly modifies tumor cell growth (21). Tegeder et al (2) reported that high concentrations of morphine inhibited tumor cell proliferation. However, other studies have drawn opposite conclusions. Lazarczyk et al (22) found that morphine triggered human glioblastoma T98G cell proliferation. Gupta et al (4) demonstrated that morphine promoted tumor neovascularization in a human breast tumor xenograft mouse model, thereby enhancing tumor progression. In the present study, it was demonstrated that morphine protected neuroblastoma cells against DKK1-induced apoptosis. The expression of DKK1 in xenografted tumors also inhibited tumor growth. However, morphine was found to significantly offset the anti-tumor effects of DKK1 in vivo. In vitro and in vivo results revealed that morphine protected neuroblastoma cells from DKK1-induced apoptosis. Furthermore, morphine had neither positive nor negative effects on the proliferation capacity of DKK1-negative neuroblastoma cells.

DKK1, a secreted protein, is known to be a negative regulator of the Wnt/ $\beta$-catenin signaling pathway (23). It was previously demonstrated that DKK1 was downregulated in human tumors, indicating that it may act as a tumor suppressor (24). In the present study, it was confirmed that DKK1 induced apoptosis in neuroblastoma cells through the Wnt/ $\beta$-catenin signaling pathway. This signaling pathway is commonly dysregulated in various cancers (25-27). LRP6 is an essential Wnt co-receptor in the Wnt/ $\beta$-catenin signaling pathway and its phosphorylation is essential for $\mathrm{Wnt} / \beta$-catenin signaling activation (28). The results of the present study additionally demonstrated a significant inhibition of LRP6 expression and phosphorylation following DKK1 treatment of SH-SY5Y cells. Moreover, in previous studies, GSK-3 was shown to be active in the absence of Wnt signaling (29), leading to the degradation of cytoplasmic $\beta$-catenin and inhibition of nuclear translocation (30). The results additionally confirmed that cytosolic and nuclear $\beta$-catenin protein expression was lost in SH-SY5Y cells following DKK1 treatment. However, morphine activated Wnt/ $\beta$-catenin signaling in DKK1-expressing SH-SY5Y cells. Neuorblastoma most commonly affects cognitive functions, including attention, psychomotor speed and memory (31). The Morris water maze (MWM), which consists of a circular pool in which mice are trained to escape from water by swimming to a hidden platform, has been described previously (32). The MWM and T-maze tests are widely used to evaluate spatial learning and memory (33). In this study, the use of the MWM and T-maze tests revealed that neuroblastoma impaired the spatial learning and memory of mice. Notably, DKK1 repaired the memory of mice by inducing the apoptosis of neuroblastoma cells. Following the administration of morphine, the effect of DKK1 was offset in the mice.
In conclusion, the results of the present study demonstrated that DKK1 inhibited SH-SY5Y cell proliferation and migration at least in part by promoting Wnt/ $\beta$-catenin signaling. Morphine was also able to protect SH-SY5Y cells against DKK1-induced apoptosis. The results of this study indicate that morphine may protect neuroblastoma cells and thus, it may be used in neuroblastoma patients.

\section{Acknowledgements}

The authors would like to thank Dr Wang Rong (Cancer Research Institute, China Medical University, Shenyang, China) for providing the pEGFP-C1-DKK1 plasmid.

\section{References}

1. Lickiss JN: Approaching cancer pain relief. Eur J Pain 5 (Suppl A): 5-14, 2001.

2. Tegeder I, Grösch S, Schmidtko A, Häussler A, Schmidt H, Niederberger E, Scholich K and Geisslinger G: G protein-independent G1 cell cycle block and apoptosis with morphine in adenocarcinoma cells: involvement of p53 phosphorylation. Cancer Res 63: 1846-1852, 2003.

3. Harimaya Y, Koizumi K, Andoh T, Nojima H, Kuraishi Y and Saiki I: Potential ability of morphine to inhibit the adhesion, invasion and metastasis of metastatic colon 26-L5 carcinoma cells. Cancer Lett 187: 121-127, 2002.

4. Gupta K, Kshirsagar S, Chang L, Schwartz R, Law PY, Yee D and Hebbel RP: Morphine stimulates angiogenesis by activating proangiogenic and survival-promoting signaling and promotes breast tumor growth. Cancer Res 62: 4491-4498, 2002.

5. Farooqui M, Li Y, Rogers T, Poonawala T, Griffin RJ, Song CW and Gupta K: COX-2 inhibitor celecoxib prevents chronic morphine-induced promotion of angiogenesis, tumour growth, metastasis and mortality, without compromising analgesia. Br J Cancer 97: 1523-1531, 2007.

6. Chen C, Farooqui M and Gupta K: Morphine stimulates vascular endothelial growth factor-like signaling in mouse retinal endothelial cells. Curr Neurovasc Res 3: 171-180, 2006

7. Katoh Y and Katoh M: Comparative genomics on DKK2 and DKK4 orthologs. Int J Mol Med 16: 477-481, 2005.

8. Scott EL and Brann DW: Estrogen regulation of Dkk1 and Wnt $/ \beta$-Catenin signaling in neurodegenerative disease. Brain Res 1514: 63-74, 2013.

9. Willert $\mathrm{K}$ and Jones KA: Wnt signaling: is the party in the nucleus? Genes Dev 20: 1394-1404, 2006.

10. Forget MA, Turcotte S, Beauseigle D, et al: The Wnt pathway regulator DKK1 is preferentially expressed in hormone-resistant breast tumours and in some common cancer types. Br J Cancer 96: 646-653, 2007.

11. Yamabuki T, Takano A, Hayama S, et al: Dikkopf-1 as a novel serologic and prognostic biomarker for lung and esophageal carcinomas. Cancer Res 67: 2517-2525, 2007.

12. Makino T, Yamasaki M, Takemasa I, et al: Dickkopf-1 expression as a marker for predicting clinical outcome in esophageal squamous cell carcinoma. Ann Surg Oncol 16: 2058-2064, 2009.

13. Patil MA, Chua MS, Pan KH, et al: An integrated data analysis approach to characterize genes highly expressed in hepatocellular carcinoma. Oncogene 24: 3737-3747, 2005.

14. Aguilera O, Fraga MF, Ballestar E, et al: Epigenetic inactivation of the Wnt antagonist DICKKOPF-1 (DKK-1) gene in human colorectal cancer. Oncogene 25: 4116-4121, 2006.

15. Yu B, Yang X, Xu Y, et al: Elevated expression of DKK1 is associated with cytoplasmic/nuclear beta-catenin accumulation and poor prognosis in hepatocellular carcinomas. J Hepato 50: 948-957, 2009.

16. Lee N, Smolarz AJ, Olson S, et al: A potential role for Dkk-1 in the pathogenesis of osteosarcoma predicts novel diagnostic and treatment strategies. Br J Cancer 97: 1552-1559, 2007

17. Weinstein JL, Katzenstein HM and Cohn SL: Advances in the diagnosis and treatment of neuroblastoma. Oncologist 8: 278-292, 2003. 
18. Maris JM,Hogarty MD, Bagatell R and Cohn SL: Neuroblastoma. Lancet 369: 2106-2120, 2007.

19. Ko YB, Kim BR, Yoon K, Choi EK, Seo SH, Lee Y, Lee MA, Yang JB, Park MS and Rho SB. WIF1 can effectively co-regulate pro-apoptotic activity through the combination with DKK1. Cell Signal 26: 2562-2572, 2014.

20. Widy-Tyszkiewicz E, Scheel-Krüger J and Christensen AV: Spatial navigation learning in spontaneously hypertensive, renal hypertensive and normotensive Wistar rats. Behav Brain Res 54: 179-185, 1993.

21. Kissin I: Scientometric assessment of drugs for chronic pain 1979-2013: rapid growth of publications, paucity of successful drugs. J Pain Res 7: 505-514, 2014.

22. Lazarczyk M, Matyja E and Lipkowski AW: A comparative study of morphine stimulation and biphalin inhibition of human glioblastoma T98G cell proliferation in vitro. Peptides 31: 1606-1612, 2010.

23. Huang Z, Li S, Song W, Li X, Li Q, Zhang Z, Han Y, Zhang X, Miao S, Du R and Wang L: Lysine-specific demethylase 1 (LSD1/KDM1A) contributes to colorectal tumorigenesis via activation of the $\mathrm{Wnt} / \beta$-catenin pathway by down-regulating Dickkopf-1 (DKK1) [corrected]. PLoS One 8: e70077, 2013.

24. Li S, Qin X, Guo X, Cui A, He Y, Wei S, Wang X and Shan B Dickkopf-1 is oncogenic and involved in invasive growth in non small cell lung cancer. PLoS One 8: e84944, 2013.

25. Sawada G, Ueo H, Matsumura T, Uchi R, Ishibashi M, Mima K, Kurashige J, Takahashi Y, Akiyoshi S, Sudo T, et al: CHD8 is an independent prognostic indicator that regulates $\mathrm{Wnt} / \mathrm{\beta}$-catenin signaling and the cell cycle in gastric cancer. Oncol Rep 30: $1137-1142,2013$.
26. Hidaka Y,Mitomi H, Saito T, Takahashi M,Lee SY, Matsumoto K, Yao T and Watanabe S: Alteration in the Wnt/ $\beta$-catenin signaling pathway in gastric neoplasias of fundic gland (chief cell predominant) type. Hum Pathol 44: 2438-2448, 2013.

27. Mo ML, Li MR, Chen Z, Liu XW, Sheng Q and Zhou HM: Inhibition of the Wnt palmitoyltransferase porcupine suppresses cell growth and downregulates the Wnt/ $\beta$-catenin pathway in gastric cancer. Oncol Lett 5: 1719-1723, 2013.

28. Zeng X, Huang H, Tamai K, Zhang X, Harada Y, Yokota C, Almeida K, Wang J, Doble B, Woodgett J, et al: Initiation of Wnt signaling: control of Wnt coreceptor Lrp6 phosphorylation/activation via frizzled, dishevelled and axin functions. Development 135: 367-375, 2008

29. Ikeda S, Kishida S, Yamamoto H, Murai H, Koyama S and Kikuchi A: Axin, a negative regulator of the Wnt signaling pathway, forms a complex with GSK-3beta and beta-catenin and promotes GSK-3beta-dependent phosphorylation of beta-catenin. EMBO J 17: 1371-1384, 1998

30. Aberle H, Bauer A, Stappert J, Kispert A and Kemler R beta-catenin is a target for the ubiquitin-proteosome pathway. EMBO J 16: 3797-3804, 1997.

31. Schleiermacher G, Janoueix-Lerosey I and Delattre O: Recent insights into the biology of neuroblastoma. Int J Cancer 135: 2249-2261, 2014.

32. Péczely L, Ollmann T, László K, et al: Effects of ventral pallidal D1 dopamine receptor activation on memory consolidation in morris water maze test. Behav Brain Res 274: 211-218, 2014.

33. Oliveira L, Graeff FG, Pereira SR, Oliveira-Silva IF, Franco GC, and Ribeiro AM: Correlations among central serotonergic parameters and age-related emotional and cognitive changes assessed through the elevated T-maze and the Morris water maze. Age (Dordr) 32: 187-196, 2010. 